\title{
Orthogonal and Straight-Line Drawings of Graphs with Succinct Representations ${ }^{\star}$
}

\author{
Ho-Lin Chen and Hsu-Chun Yen \\ Dept. of Electrical Engineering, National Taiwan University \\ Taipei, Taiwan, R. O. C. \\ yen@cc.ee.ntu.edu.tw
}

The concept of a hierarchical design has played an important role in various areas of computer science and engineering, including software engineering, CAD, among others. Graphs with hierarchical structures, which are capable of describing large-scale regular structures in a succinct manner, have naturally become an interesting and important modeling tool for facilitating such a hierarchical design. In the literature, a number of succinct models for representing graphs with hierarchical structures have been proposed. See, e.g., [4,5]. In each of such hierarchical graph models, a succinct description is capable of representing a graph (which can be thought of as the expansion of the description) whose size is exponential in the length of the description.

A hierarchical graph $[4,5] \Gamma=\left(G_{1}, \ldots, G_{k}\right)$ of depth $k$ contains $k$ cells $G_{1}, \ldots, G_{k}$, each of which is a graph consisting of two types of vertices, namely, terminals and nonterminals. Intuitively speaking, nonterminals are those that are going to be replaced by cells of smaller indices during the expansion process. Suppose $G_{i}=\left(V_{i}, E_{i}\right), 1 \leq i<k$, where $V_{i}$ and $E_{i}$ are the sets of vertices and edges of $G_{i}$, respectively. For each $G_{i}$, there is a pin assignment function $\rho_{i}:\left\{1, \ldots, d_{i}\right\} \rightarrow V_{i}$ (where $d_{i}$ is a positive integer), which specifies the way vertices are connected to the upper layer. The $d_{i}$ is called the degree of $G_{i}$, and each of the vertices in $\rho_{i}\left(\left\{1, \ldots, d_{i}\right\}\right)$ is called a frontier vertex (f-vertex, for short). Each nonterminal inside $G_{i}$ is specified as $\left(m, G_{j}\right)$ where $m$ (an integer) represents the unique name of the nonterminal, and $G_{j}, 1 \leq j<i$, denotes the type of the nonterminal. A nonterminal of type $G_{j}$ has degree $d_{j}$, and each incident edge is labeled by a unique integer in $\left\{1, \ldots, d_{j}\right\}$. The expansion of $G_{i}$, denoted by $E\left(G_{i}\right)$, is obtained by expanding all of its subcells $G_{1}, \ldots, G_{i-1}$ recursively, and then replacing each nonterminal of type $G_{j}(1 \leq j<i)$ inside $G_{i}$ by a copy of $E\left(G_{j}\right)$ in such a way that each incident edge labeled $l\left(1 \leq l \leq d_{j}\right)$ is connected to the f-vertex $\rho_{j}(l)$. The expansion of a hierarchical graph $\Gamma=\left(G_{1}, \ldots, G_{k}\right)$, denoted by $E(\Gamma)$, is defined to be $E\left(G_{k}\right)$.

In this research, our goal is to 'draw' hierarchical planar graphs on grids in the styles of straight-line drawing (i.e., each edge is a straight-line segment) and orthogonal drawing (i.e., each edge is a chain of horizontal and vertical segments) [2]. Since the size of the expansion of a hierarchical graph can be exponential in the length of its succinct representation, care must be taken when defining

* This work was supported in part by the National Science Council of the Republic of China under Grant NSC-88-2815-C-002-002-E. 
the output of a graph drawing algorithm. In our setting, the outputs of our drawing algorithms are succinct representations of drawings and not of drawings themselves. (That is, we require that the drawing of a hierarchical graph be expressible succinctly as well.) To this end, the drawings of two copies of the same cell must be 'identical' in the sense that one drawing can be obtained by the operations of flipping with respect to the y-axis and/or rotations of $90^{\circ}, 180^{\circ}$ or $270^{\circ}$ from the other. A hierarchical graph is said to have a planar straightline (resp., orthogonal) grid drawing if its expansion can be drawn on grids in a straight-line (resp., orthogonal) fashion without edge crossings subject to the above constraints regarding copies of each cell. It should be noted that our hierarchical graph model differs from that of 'hierarchical graphs' used in, e.g., [3]. The latter refers to graphs whose vertices are assigned to layers, and the so-called 'hierarchical drawing' is to place all the vertices of a hierarchical graph on a set of equally-spaced horizontal lines.

The main contributions of this paper include the design and analysis of orthogonal and straight-line drawing algorithms which operate on the succinct descriptions of hierarchical graphs directly, and output succinct representations of drawings. (For related results, the reader is referred to [1].) We do not require that the hierarchical graphs be expanded in order for our algorithms to work. To the best of our knowledge, conventional graph drawing algorithms operate only on completely specified graphs. For hierarchical graphs $\left(G_{1}, \ldots, G_{k}\right)$ with the number of outgoing connections in each $G_{i}$ bounded by 2 , we derive a planar straight-line grid drawing algorithm which runs in time $\sum_{i=1}^{k}\left|G_{i}\right|$, where $\left|G_{i}\right|$ denotes the number of vertices in $G_{i}$. (Notice that $\sum_{i=1}^{k}\left|G_{i}\right|$ is linear in the size of the succinct representation.) The drawing area of the expanded graph is bounded by $O\left(2^{3 k} \prod_{i=1}^{k}\left(\left|G_{i}\right|-2\right)^{6}\right)$. For orthogonal grid drawings of hierarchical planar graphs, we present an algorithm which runs in $O\left(\sum_{i=1}^{k}\left|G_{i}\right|^{2}\right)$ time, provided that the input graph is 2 -connected (i.e., a graph which remains connected even if a single vertex is removed). The drawing area and the total number of bends of the expanded graph are bounded by $O\left(n^{2}\right)$ and $\left(\max _{i=1, \ldots, k}\left\{\left|G_{i}\right|\right\}\right)^{k}$, respectively, where $n$ is the number of vertices in the expanded graph. Our algorithm can also be used to report whether the input graph exhibits a planar straight-line (or orthogonal) grid drawing.

\section{References}

1. F. Brandenburg, Designing Graph Drawings by Layout Graph Grammars, Graph Drawing'94, LNCS 894, pp. 416-427, 1994.

2. G. Di Battista, P. Eades, R. Tamassia, and I. Tollis, Graph Drawing: Algorithms for the Visualization of Graphs, Prentice-Hall, 1999.

3. P. Eades, X. Lin, and R. Tamassia, An Algorithm for Drawing a Hierarchical Graph, Internat. J. Comput. Geom. Appl., Vol. 6, pp. 145-156, 1996.

4. T. Lengauer, Efficient Algorithms for Finding Minimum Spanning Forests of Hierarchically Defined Graphs, J. Algorithms, Vol. 8, pp. 260-284, 1987.

5. T. Lengauer, Hierarchical Planarity Testing Algorithms, J. ACM, Vol. 36, No. 3, pp. 474-509, 1989. 